\title{
Reconfiguration des pratiques touristiques de haute intensité en temps de pandémie : l'impact des contraintes à la mobilité dans l'Est-du-Québec
} Reconfiguration of High-intensity Tourism Practices in Times of Pandemic: The Impact of Mobility Constraints in Eastern Quebec

\section{Luc Renaud et Dominic Lapointe}

\section{(2) OpenEdition Journals}

Édition électronique

URL : https://journals.openedition.org/tourisme/3930

DOI : 10.4000/tourisme.3930

ISSN : 2492-7503

\section{Éditeur}

Association Mondes du tourisme

\section{Référence électronique}

Luc Renaud et Dominic Lapointe, «Reconfiguration des pratiques touristiques de haute intensité en temps de pandémie : l'impact des contraintes à la mobilité dans l'Est-du-Québec », Mondes du Tourisme [En ligne], 20 | 2021, mis en ligne le 15 décembre 2021, consulté le 17 décembre 2021. URL: http://journals.openedition.org/tourisme/3930 ; DOI : https://doi.org/10.4000/tourisme.3930

Ce document a été généré automatiquement le 17 décembre 2021.

\section{cc) (†)}

Mondes du tourisme est mis à disposition selon les termes de la licence Creative Commons Attribution - Pas d'Utilisation Commerciale - Pas de Modification 4.0 International. 


\section{Reconfiguration des pratiques touristiques de haute intensité en temps de pandémie : l'impact des contraintes à la mobilité dans l'Est- du-Québec}

Reconfiguration of High-intensity Tourism Practices in Times of Pandemic: The Impact of Mobility Constraints in Eastern Quebec

Luc Renaud et Dominic Lapointe

\section{Introduction}

1 La transformation des mobilités territoriales liée à la pandémie de COVID-19, observée à l'été 2020 , a eu des effets importants sur les pratiques touristiques de l'Est-du-Québec ( $c f$. figure 1) et a soulevé de nouveaux défis en termes de gestion de l'espace de vie de ses habitants et de leurs pratiques territoriales. À l'aube de la nouvelle saison touristique estivale 2021, alors qu'il apparaît de plus en plus clairement que la mobilité touristique « habituelle » risque d'être une nouvelle fois compromise par la persistance de la pandémie, les acteurs du tourisme de l'Est-du-Québec se préparent à une autre saison hors du commun. En effet, l'engouement que cette région a connu en raison de la fermeture des frontières des États-Unis et de l'Est du Canada a induit de nouveaux flux touristiques qui ont affecté le tourisme. Bien que parfois problématique, ce nouvel attrait pour une région périphérique est aussi porteur d'opportunités de développement touristique et de prise en charge territoriale.

2 Par cette contribution, nous cherchons à montrer comment les nouvelles mobilités pandémiques régionales ont affecté les pratiques touristiques d'un espace périphérique. Notre démarche s'appuie sur des entretiens semi-dirigés, des 
observations participantes et l'analyse de contenu de sources médiatiques. Après une brève discussion des concepts de mobilité et de proximité utiles à notre analyse, nous dresserons un portrait global de la saison touristique estivale impactée par la pandémie dans les deux grands centres urbains québécois, Montréal et Québec, ainsi que dans la région de la Gaspésie-Îles-de-la-Madeleine dans l'Est-du-Québec. Cette vue d'ensemble permettra ensuite de mettre en évidence la transformation des flux touristiques induits par la pandémie afin d'aborder la question des impacts territoriaux et de la transformation des imaginaires centre-périphéries. Finalement, nous poserons la question du développement touristique et de la reconfiguration des territorialités périphériques en nous appuyant sur les notions liées au capital de mobilité et de proximité.

Figure 1. Est-du-Québec

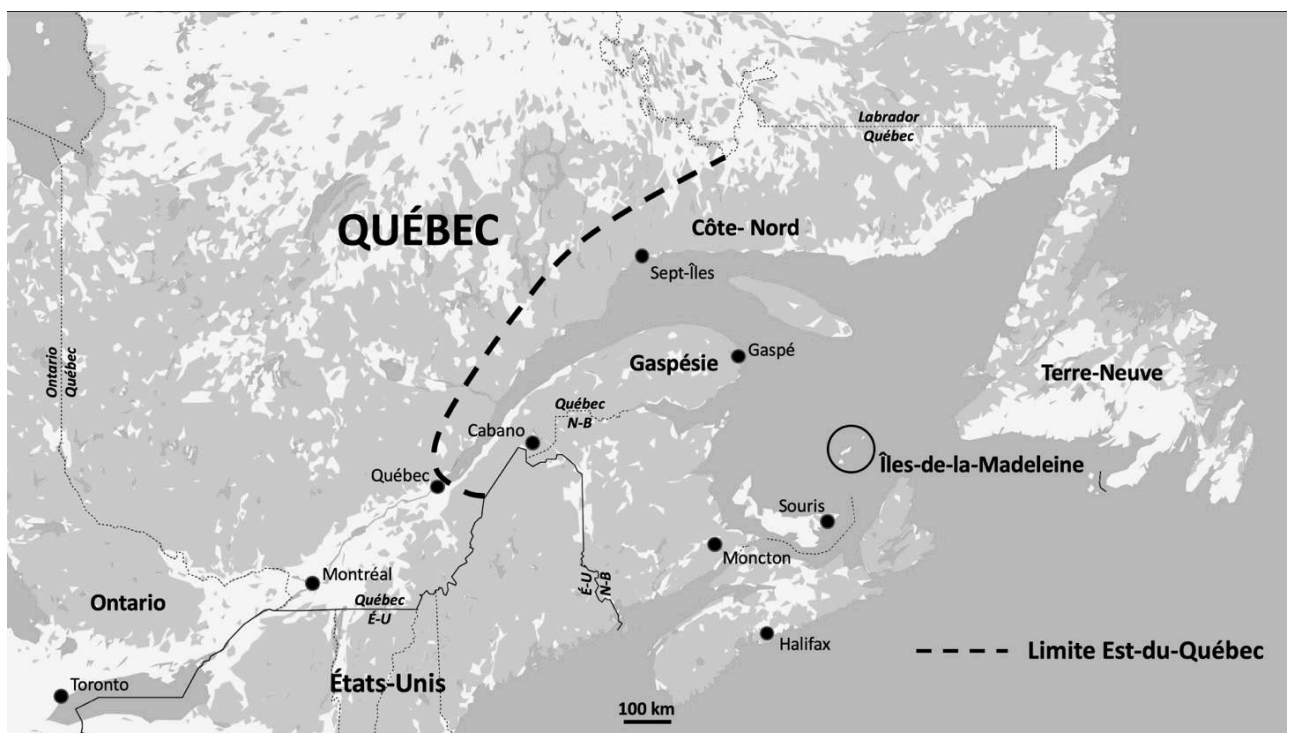

Conception : Luc Renaud et Dominic Lapointe

\section{Mobilité/proximité}

C'est le 13 mars 2020 que le gouvernement québécois annonce le début des mesures de confinement et le rapatriement des Canadiens à l'étranger suite aux premières éclosions de COVID-19 sur le territoire. Cinq jours plus tard, le gouvernement canadien, de concert avec les autorités américaines, décide de fermer la frontière et, pour la première fois de l'histoire, la mobilité touristique entre les deux pays est entravée. À cette fermeture - toujours en vigueur lors de la rédaction de la dernière version de ce texte - s'est également ajoutée la fermeture des frontières des provinces maritimes canadiennes qui ont formé ensemble une « bulle » sanitaire de l'Atlantique. À l'aube de la saison touristique 2020, une toute nouvelle configuration des flux touristiques est apparue, caractérisée par une mobilité globale ou transnationale inexistante et une mobilité régionale contrainte. L'été de la COVID-19 au Québec allait dès lors être défini par une dynamique localiste alors que la population se voyait forcée de demeurer sur le territoire provincial. 
4 Avant la pandémie, la pratique touristique estivale typique au Québec est représentative des sociétés qui empruntent à la fois au globalisme et au localisme au sens de la typologie proposée par Ferreira et al. (2017). Le globalisme fait référence à la mobilité en tant que capital disponible. Appliqué aux pratiques touristiques, ce type de capital permet de valoriser l'accès aux ressources touristiques - territoires, populations et culture - par le biais de déplacements à grande échelle qui reposent sur d'importantes infrastructures de transport. Partie intégrante du tourisme transnational, ce type de capital permet aux touristes de se connecter à une variété de ressources touristiques rendues disponibles à travers des réseaux qui peuvent être largement indépendants des territoires. Par exemple, les pratiques événementielles touristiques urbaines, telles que les grands festivals de musique, peuvent être reproduites d'un lieu à l'autre de façon pratiquement identique. Ce type de capital contribue cependant à affaiblir les avantages comparatifs, voire concurrentiels, d'un territoire étant donné la nature transférable de certaines de ces pratiques touristiques. À titre d'exemple, les dirigeants de la Formule 1 ont simplement transféré le Grand Prix du Canada en Turquie pour l'édition 2021.

5 De son côté, le localisme fait référence à la notion de proximité en tant que capital disponible. Appliqué aux pratiques touristiques, il s'agit de permettre une valorisation des ressources touristiques grâce aux liens de proximité tissés entre les individus et le territoire afin de répondre à leurs besoins et leurs aspirations, et ce, sans devoir dépendre d'importantes infrastructures de transport. Ce type de capital permet une adhésion à l'espace de vie, ainsi qu'à des pratiques sociales qui s'y rattachent et qui permettent de valoriser la cohésion entre les individus et leur territoire. Ultimement, la notion de proximité favorise non seulement le savoir et les compétences locales, mais elle donne aussi aux populations un levier pour développer un sens d'appartenance à leur environnement.

6 Cette analyse "globale/locale » doit tenir compte du tiraillement incessant entre la force du capital liée à la mobilité et celle du capital lié à la proximité, dans lequel s'insèrent les différents espaces touristiques, ceux-ci étant par ailleurs soumis aux forces du «Worldmaking» (Hollinshead et al., 2009). Ce concept, que nous traduisons par la "globotouristification» des espaces de vie, soutient qu'à travers l'exercice du pouvoir de la part de groupes d'acteurs, il y a l'imposition hégémonique - consciente ou inconsciente - d'une trame narrative idéologique de domination (Lapointe et al., 2018; Fletcher et al., 2020). Les espaces du tourisme du Québec n'y échappent pas. Ces « jeux » de tension impliquent des relations de pouvoir entre les acteurs tenants du capital lié à la mobilité et ceux rattachés au capital de proximité, dans un contexte où les premiers contribuent à une production de l'espace touristique qui prive les populations réceptrices d'une capacité de prise en charge de leur territoire. En d'autres mots, la globotouristification va logiquement tirer profit du globalisme et sa rencontre avec le localisme va induire des espaces de résistance de la part des populations locales pour faire face aux forces normalisatrices et homogénéisatrices de la mobilité globale (Renaud, 2020a).

7 Une société « globotouristifiée » va dès lors représenter les caractéristiques d'un espace de vie liquide au sens de Bauman (2000) ; c'est-à-dire un espace où les repères locaux s'effritent, où règne la consommation dans l'univers du jetable, où se développe une hypermobilité axée sur l'efficacité et la marchandisation des ressources matérielles et imaginaires des sociétés réceptrices. Au sein de cette société fluide, la proximité 
devient un capital non disponible (Ferreira et al., 2017) dans une dynamique d'altérisation du tourisme vis-à-vis des espaces où il prend place (Lapointe, 2020), à l'exemple des grandes destinations transnationales telles que Montréal. Ici, la fluidité est donc considérée comme un état transitoire entre une valorisation de la mobilité au détriment de la proximité. Toutefois, même si elle est porteuse de problématiques anthropocéniques, une société fluide n'est pas moins souhaitable, dans la perspective du tourisme, que son opposé sociétal de type stagnant, la différenciation et les avantages comparatifs se trouvant à l'intersection des deux formes sociétales.

En effet, une situation où la mobilité devient un capital indisponible (Ferreira et al., 2017) - ce que la COVID-19 a imposé aux sociétés touchées par la pandémie - n'est pas plus supportable. Les populations stagnantes attendent désespérément une reprise de la mobilité transnationale au risque de provoquer un effet indésirable de "Revenge Travelling", ou voyage revanchard, lié à une envie de mobilité refoulée par la pandémie (Shadel, 2020). Entre une société fluide axée sur le globalisme et une société stagnante se repliant sur le localisme, il s'agit de trouver une zone durable au sein de laquelle un espace de vie peut entrer en relation avec l'objet «tourisme » de façon à satisfaire les besoins de développement. Pour se faire, les dynamiques socioterritoriales de coopération, d'adaptation ou de résistance face au tourisme sont à considérer.

Envisagés selon cette perspective, le globalisme et le localisme ne s'insèrent donc pas dans une relation d'exclusion. En effet, au Québec, les milieux urbains plus "globaux » comme les milieux périphériques plus «locaux " peuvent tirer profit des capitaux de mobilité/proximité. À ce titre, chaque espace de vie qui entre en relation avec le tourisme doit déterminer un dosage adéquat en réponse aux orientations prises par les instances de gouvernance ou par les différents groupes d'acteurs selon le développement touristique souhaité. Il est toutefois intéressant de constater qu'au cours de la dernière année, la pandémie de COVID-19 a mis en lumière la capacité différenciée de résilience associée à ces types de capitaux. Elle a permis d'observer comment les espaces de vie ont su s'adapter aux transformations des mobilités apportées par une situation de crise selon une valorisation de la mobilité ou de la proximité.

10 Alors que le tourisme globaliste tend à s'inscrire dans l'hypermobilité ou la haute mobilité (Viry et Kaufmann, 2015) - longue distance, haute fréquence et haute vitesse -, celui-ci a largement démontré sa faible capacité de résilience étant donné sa dépendance à la mobilité, laquelle s'avère aisément perturbable. Les attaques terroristes, l'instabilité géopolitique, les événements climatiques ou géologiques et, maintenant, la crise sanitaire mondiale sont autant de facteurs mettant en relief le caractère fragile de la mobilité en tant que capital disponible permettant un accès aux ressources territoriales. Cela est d'autant plus vrai lors de la présente crise sanitaire où, à la différence des crises précédentes, le contrôle de la mobilité et de l'immobilité se négocie à la fois à destination, mais aussi dans les pôles émetteurs, appelant une coordination sensible entre des contextes hétérogènes. À l'instar des réflexions portant sur la capacité de résilience liée à la proximité (mobilité lente et immobilité) comme capital social, les nouvelles dynamiques touristiques provoquées par la COVID appellent également à questionner la notion de localisme qui teinte certaines pratiques touristiques, tel le «slow tourism » jusqu'à tout récemment cantonné à un segment de niche (Caffyn, 2012; Moira et al., 2017). Ainsi, l'analyse de la reconfiguration des flux touristiques au Québec que nous proposons témoigne du degré de résilience important 
du capital de proximité à l'échelle régionale et locale à un moment où la mobilité transnationale, elle, est mise en arrêt.

\section{Bilan contrasté centre-périphérie}

11 Le changement majeur de l'été 2020 a été le déplacement des activités touristiques vers les périphéries des deux grands centres urbains de Montréal et de la ville de Québec, privés du tourisme transnational et abandonnés par leurs résidents. Alors que la population venait de subir un confinement sévère et que les activités événementielles qui caractérisent habituellement le tourisme urbain estival étaient pour la plupart annulées, les citadins se sont tournés en masse vers les régions. La figure 2 montre les principaux flux touristiques observables durant l'été 2020. C'est Montréal qui a subi les pires contrecoups de la pandémie alors que son industrie s'est littéralement effondrée ${ }^{1}$ malgré l'appel à visiter Montréal fait aux Montréalais et à l'ensemble des Québécois par la mairesse et Tourisme Montréal pour pallier l'absence des touristes transnationaux (Girard, 2020 ; Tourisme Montréal, 2020). En effet, en 2019, Montréal a attiré 3 millions de touristes étrangers. La ville a donc été particulièrement affectée par l'effondrement des arrivées à la frontière en 2020 par rapport à $2019(-97,8 \%)$ ainsi qu'à l'aéroport international de Montréal (-94,5\%), de l'occupation des hôtels $(-70 \%)$ et de la fréquentation des principaux attraits touristiques $(-75 \%)$. L'absence de touristes étrangers n'explique pas à elle seule cet effondrement, car ils ne représentent que près de $25 \%$ des visiteurs. Les Québécois ont également déserté la métropole.

12 La ville de Québec, inscrite au patrimoine mondial de l'Unesco a, quant à elle, un peu mieux tiré son épingle du jeu. Comparativement à la métropole, les impacts subis par rapport à 2019 ont été moins importants. Il n'en demeure pas moins que les indicateurs de performance sont relativement négatifs ${ }^{2}$ au niveau de la fréquentation de l'aéroport international Jean-Lesage $(-71,2 \%)$, de l'occupation des hôtels $(-57,7 \%)$ et de la fréquentation des attraits touristiques $(-64,9 \%)$. Alors que les visiteurs internationaux brillent par leur absence, les acteurs du tourisme au Québec doivent donc s'appuyer sur le tourisme domestique pour sauver les meubles, mais force est de constater que les Québécois n'ont pas choisi ces grandes villes pour les vacances estivales.

Si le tourisme local ne semble pas avoir été en mesure de sauver la saison touristique à Montréal et à Québec, il en va tout autrement des régions périphériques. Le 11 septembre 2020, un quotidien de la ville de Québec coiffait un article du titre suivant: "Tourisme: l'été de rêve se poursuit en Gaspésie ${ }^{3}$ ». En effet, les régions périphériques, dont font partie la Gaspésie et les îles de la Madeleine, ont été témoin d'un engouement certain alors qu'affluaient en grand nombre les touristes. Le constat général montre que, malgré une baisse de la fréquentation par rapport à 2019, l'été de la COVID-19 n'a pas été la catastrophe qui s'annonçait au printemps alors qu'au pire de la première vague d'infections, les autorités gouvernementales avaient interdit les mobilités non essentielles vers les régions périphériques en érigeant des barrages routiers. 
Figure 2. Reconfiguration territoriale touristique suite à la pandémie de COVID-19

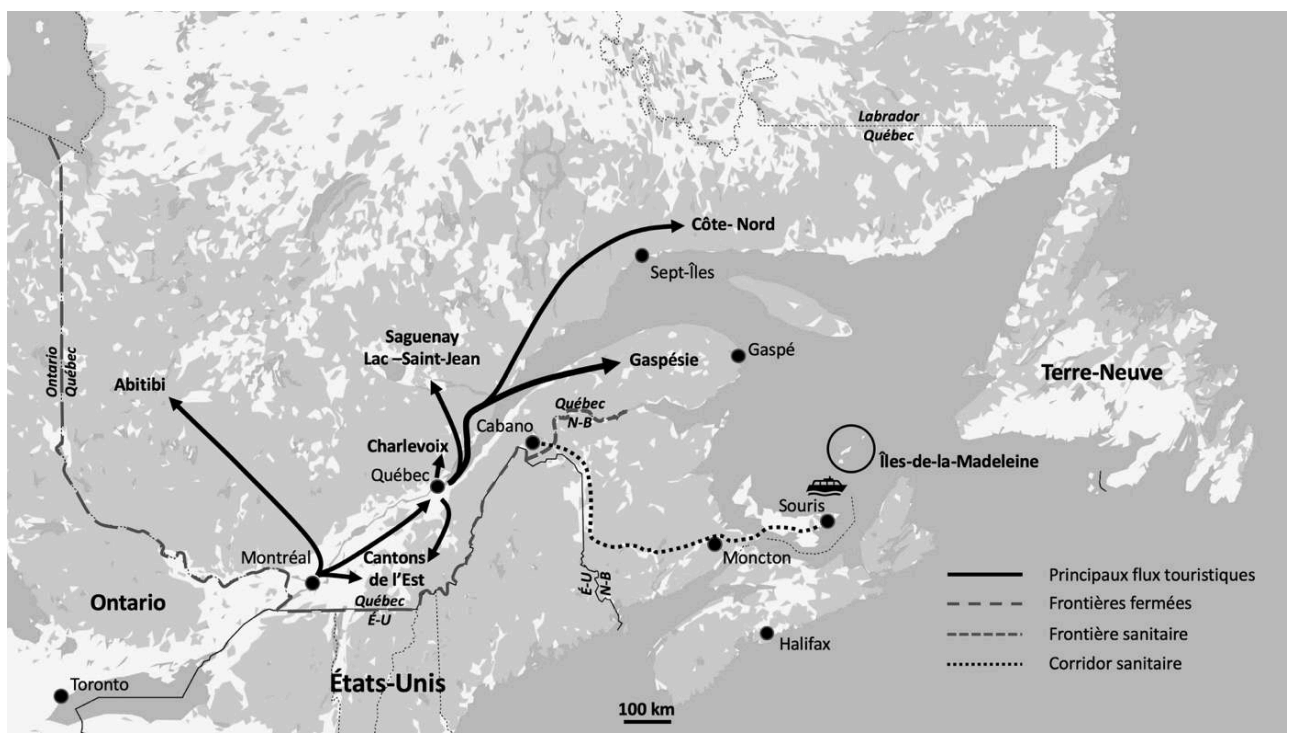

Conception : Luc Renaud et Dominic Lapointe positif de la saison $2020^{4}$, et ce, même s'il est difficile de comparer cette saison avec les indicateurs habituels. Alors que le taux d'occupation du secteur hôtelier en juillet et août a reculé de 2,2 points par rapport à 2019 et que les sites de camping ont été occupés à pleine capacité, la nature de l'offre d'hébergement était très différente en raison de la pandémie. Le fait que les auberges de jeunesse et leurs dortoirs aient été fermés et que $19 \%$ des campings aient fermé leurs blocs sanitaires et n'accueillaient que les campeurs autonomes a pu favoriser une plus grande occupation des hôtels. Si l'impact des fermetures non hôtelières est difficile à mesurer ${ }^{5}$ et que le taux d'occupation réel reste inconnu, il n'en demeure pas moins que la saison estivale a répondu à plus de $90 \%$ à l'attente des entreprises membres de l'ATR. Alors que la Gaspésie pousse un soupir de soulagement lorsque l'accès à son territoire a de nouveau été permis le 18 mai 2020, lui permettant d'envisager l'accueil des visiteurs dès le début de l'été, il en va tout autrement pour les îles de la Madeleine.

En effet, la limitation de la capacité du traversier et la limite de visiteurs fixée à 35000 durant l'été 2020 ont porté un coup dur à l'économie locale de l'archipel, situé en plein golfe du Saint-Laurent. Finalement, l'archipel, pour lequel le tourisme est le deuxième secteur économique le plus important, a subi, selon l'ATR locale ${ }^{6}$, une baisse de fréquentation de $50 \%$ durant les mois d'été, en comparaison à l'été 2019. Bien que mitigé, le bilan présenté montre que la baisse de fréquentation s'explique par les contraintes de transport liées à la COVID et non par un manque d'engouement de la part des touristes. En effet, les places disponibles ont rapidement été comblées et la capacité permise a été occupée à $100 \%$. De plus, autre fait notable, la pandémie et les difficultés d'accès n'ont pas empêché de nouveaux visiteurs de découvrir la destination car, selon l'ATR locale, 64 \% des visiteurs de l'été 2020 en étaient à leur première visite.

Il est important de noter que cet engouement ne s'est pas limité à l'Est-du-Québec, mais s'est également manifesté dans les autres régions périphériques. Une enquête menée l'été dernier par le ministère du Tourisme ${ }^{7}$ a révélé que les entreprises touristiques s'étant déclarées " plutôt satisfaites » de leur été 2020 étaient pour la plupart situées en 
périphérie des grands centres urbains ainsi que dans les régions touristiques de l'Abitibi, de Charlevoix, des Cantons-de-l'Est, de Saguenay-Lac-Saint-Jean, de la Gaspésie et de la Côte-Nord. De plus, ce sont des secteurs comme l'agrotourisme et les sites de camping qui se sont montrés les plus satisfaits de la saison, témoignant d'un meilleur bilan que les espaces urbains.

\section{Dynamique des flux touristiques}

17 Une fois ce constat effectué, nous proposons de mettre en lumière les effets induits par certains mécanismes territoriaux dans la distribution des flux touristiques centre/ périphérie lors de la première vague de la pandémie. Nous nous intéresserons d'abord à l'impact de la fermeture des frontières, puis à la mise en place d'un corridor sanitaire pour permettre aux touristes d'accéder aux îles de la Madeleine.

Tout d'abord, la figure 2 montre les axes de mobilité touristique qui sont apparus durant l'été 2020, alors qu'une grande partie des touristes des grands centres urbains s'est dirigée vers les destinations périphériques. La raison principale, à l'origine de cette dynamique inédite, est la fermeture de la frontière américaine et l'instauration de la bulle sanitaire des provinces maritimes, comme nous l'avons évoqué plus haut. Toujours en vigueur au moment d'écrire ces lignes, cette première mesure a eu pour effet de rendre impossible tout séjour touristique chez le voisin du sud, alors que plusieurs destinations vacances de la côte est des États-Unis sont très prisées durant la saison estivale. La deuxième mesure a aussi privé les Québécois d'une destination également très populaire durant la saison estivale. En l'espace d'une semaine, une grande partie de l'offre balnéaire de l'est du continent américain a donc disparu, ne laissant d'autres choix aux Québécois, déconfinés de leur demeure, mais confinés à leur territoire, que de se rabattre sur la Gaspésie et les îles de la Madeleine pour accéder à la " mer».

Si la Gaspésie demeure facile d'accès, malgré l'éloignement des grands centres, il en va tout autrement de l'archipel madelinot en raison de la fermeture de la frontière provinciale avec le Nouveau-Brunswick. Dès la fin mars, l'Est-du-Québec est fermé aux voyages non essentiels et la décision de rouvrir éventuellement cette région revient aux autorités provinciales québécoises. C'est ce qui se produit le 18 mai 2020, laissant entrevoir une possibilité pour la Gaspésie de recevoir des visiteurs. Or, le 12 mai, la décision du gouvernement néo-brunswickois de ne pas rouvrir les frontières avec le Québec durant tout l'été isole les îles de la Madeleine et provoque une onde de choc dans l'industrie touristique. Alors que les vols à destination de l'archipel sont déjà suspendus depuis le $1^{\mathrm{er}}$ avril, l'annonce du maintien des restrictions de mobilité signifiait la perte de la saison touristique, car le seul moyen de se rendre dans l'archipel est d'emprunter le traversier au départ du port de Souris à l'île-du-Prince-Édouard (cf. figure 2). Face à cette situation, le député provincial des îles de la Madeleine fait officiellement la demande de la mise en place d'un corridor sanitaire qui permettrait aux touristes de s'y rendre, demande qui a été acceptée à la mi-juin. Le corridor sanitaire oblige les vacanciers à effectuer d'une traite, sans arrêt ni escale, le trajet entre la petite ville de Cabano, au Québec, dès lors transformée en base d'attente et envahie par les centaines de visiteurs en transit, et le port de Souris, à l'île-du-PrinceÉdouard. D'une durée de $7 \mathrm{~h} 30^{8}$, le trajet qu'il faut parfois effectuer de nuit pour avoir le traversier du matin, s'est révélé être un défi important pour les vacanciers, notamment 
en raison de la fatigue et des risques élevés de collision avec la faune sauvage durant les trajets nocturnes.

Si plusieurs régions du Québec ont noté des modulations dans les déplacements des touristes en lien avec la restructuration des flux, c'est l'Est-du-Québec qui a observé les changements les plus notables, son territoire devenant particulièrement prisé tout au long de l'été 2020. De fait, la popularité de la région a eu des impacts en termes de gestion territoriale, avec l'arrivée massive de touristes visitant pour la première fois la région, et a également mis en évidence le décalage des imaginaires territoriaux entre les visiteurs et les locaux - ces derniers ayant plutôt décidé de rester à la maison en raison de la pandémie. Aux îles de la Madeleine, c'est une réduction de $70 \%$ des déplacements des Madelinots vers le continent qui a été observée; en bref, la population a peu quitté l'archipel, sauf pour les déplacements essentiels. À la lumière de ces observations, nous pouvons considérer l'été 2020 comme un été tout à fait inédit dans l'histoire du tourisme québécois, un été au cours duquel les grandes places urbaines étaient désertes tandis que les multiples plages en périphérie étaient bondées.

\section{Impacts territoriaux et de la transformation des imaginaires centre-périphéries}

«Été de rêve» en Gaspésie pour certains; «sauvetage de la saison » aux îles de la Madeleine pour d'autres... Si ces qualificatifs sont certes représentatifs de l'été 2020, la nouvelle restructuration des flux touristiques a aussi apporté son lot de défis en termes de gestion territoriale, soulevant entre autres la problématique de la gouvernance centre/périphérie. À ces défis s'ajoutent également des tensions liées aux usages des espaces de vie induites par le décalage des imaginaires respectifs des touristes et de la population locale.

Même si ce sentiment n'est pas généralisé, c'est l'impression d'une invasion massive du domaine littoral qui a habité une frange de la population gaspésienne, laquelle a l'habitude de fréquenter cet environnement tout au long de l'année. Le constat est brutal : plages souillées, végétation côtière détruite et équipements abandonnés. Pour les municipalités aux prises avec ce problème, le défi s'est avéré double. D'une part, la disparition d'une grande partie de l'offre d'hébergement non hôtelier et l'élimination des sites de campings "mous » dédiés aux campeurs ne possédant pas d'installations sanitaires (principalement les utilisateurs de tentes) ont fait que bien des touristes arrivant dans la région ont tout simplement été incapables de trouver des sites de camping officiels. Ces touristes, du moins à la lumière de constats établis sur le terrain, en étaient souvent à leur première expérience de camping et ont dû se rabattre sur les plages de la région. C'est dans ce contexte que des manques de civisme ont été observés. D'autre part, étant donné que la gestion de la zone de marnage du littoral au Québec relève de la juridiction provinciale, les municipalités ont été dans l'impossibilité de limiter l'accès aux plages.

La saison touristique hors-norme de l'été 2020 se caractérise notamment par une concentration de la fréquentation des sites, doublée d'un redéploiement des imaginaires touristiques en lien avec le littoral gaspésien et madelinot. Tout d'abord, selon les associations touristiques locales, avant la pandémie, ces deux destinations étaient déjà des espaces touristiques, principalement fréquentés par un tourisme domestique, et ce à plus de $80 \%$. Alors que les îles de la Madeleine représentent une 
destination en soi, avec un séjour moyen de neuf jours et un fort imaginaire balnéaire associé, lié à ses plages, ses châteaux de sable et les différentes activités nautiques que l'on peut y pratiquer, la Gaspésie a une trajectoire touristique bien différente. En effet, un voyage en Gaspésie se définit le plus souvent selon un trajet qui prend la forme d'un tour, idée évidemment favorisée par la géomorphologie de la péninsule avec ses littoraux nord et sud et son massif montagneux, les Chic-Chocs, lequel n'est traversé que de trois routes transversales. Ajoutons à cela que ce «tour » a été popularisé dans l'après-guerre par les conducteurs de l'American Automobile Association (AAA) et qu'il est associé à un imaginaire des grands espaces, de la nature sauvage et d'une culture locale ancrée dans une relation unique entre la mer et la terre, entre la pêche et l'agriculture. Cet imaginaire perdure : de nos jours encore, la Gaspésie est abordée dans la perspective d'en faire un tour; ses paysages font partie de la promotion de la destination Québec, avec des icônes, tels le rocher Percé, la pointe de Forillon, les caribous du mont Jacques-Cartier ; ses saveurs, parmi lesquelles le homard et le crabe des neiges, mais aussi ses multiples microbrasseries et fumoirs à poisson sont recherchées. À cet imaginaire se greffent des kilomètres de plages "sauvages ", peu aménagées et plus propices à la promenade qu'à la baignade. Ainsi, malgré l'omniprésence de la "mer », la Gaspésie d'avant l'été 2020 n'était pas rattachée à un imaginaire balnéaire fort.

Le déconfinement graduel, survenu au Québec entre le 18 mai 2020 et la fin du mois de juin 2020, a mis fin à l'immobilité forcée pour laisser place à une mobilité tronquée et restreinte au territoire provincial. Les déplacements en dehors de la province étaient déconseillés et soumis à un haut niveau de contraintes, dont l'instauration d'une quarantaine fermée de quatorze jours. Néanmoins, ce retour de la mobilité doublé d'un été caniculaire - Montréal ayant vécu, de manière inhabituelle, sa première canicule fin mai avec des températures records de 33,1 degrés Celsius le 27 mai 2020 - a créé une ruée vers la nature. La Société des établissements de plein air du Québec (SEPAQ) enregistre d'ailleurs en 2020 une année record'. Ainsi, à l'arrivée de la saison estivale, les habitués des stations balnéaires de la côte est américaine et des Maritimes se replient sur les plages de l'Est-du-Québec, apportant avec eux les pratiques et les imaginaires associés à leurs voyages habituels. Tel qu'évoqué plus haut, les plages de Gaspésie où ces nouveaux visiteurs débarquent sont, elles, bien peu aménagées : elles sont le lieu de promenades, d'observations de la nature, de socialisation de faible intensité, par exemple les feux de camp intimes en soirée malgré des interdictions ponctuelles, la cueillette de mollusques, les baignades « audacieuses » en eaux froides.

Cette fréquentation relativement douce de la plage par les visiteurs familiers se superpose à une utilisation similaire par les résidents, lesquels ont un fort sentiment d'appartenance et d'appropriation vis-à-vis de l'espace plage. Ainsi, l'imaginaire communément répandu de la plage comme espace de forte sociabilité et de loisirs, incluant la baignade, des aménagements, des services et des commerces, n'est pas dominant en Gaspésie. De plus, on n'y retrouve aucunement la densité de fréquentation qui est également souvent associée à la pratique et à l'imaginaire balnéaires. C'est ainsi qu'à l'été 2020, des imaginaires et des usages conflictuels se sont superposés sur les plages gaspésiennes, où la tranquillité des plages non aménagées s'est vue investie par des visiteurs aux pratiques balnéaires plus proches des stations de la côte atlantique, avec sports de plage, cuisines extérieures et chaises de parterre. À ceci s'est ajouté un camping sauvage imprévu, en partie dû à l'offre limitée de campings aménagés. Assez rapidement, l'enjeu de l'absence d'aménagements d'accueil s'est fait sentir, plus 
particulièrement en ce qui a trait aux conditions d'hygiène. Cette situation a généré un conflit dans lequel l'imaginaire de la plage non aménagée propre aux résidents, mais aussi aux visiteurs habitués des littoraux gaspésiens, a été heurté par l'appropriation balnéaire de visiteurs majoritairement urbains en quête d'espaces festifs.

Du côté des îles de la Madeleine, l'imaginaire balnéaire est déjà présent. En effet, l'insularité, doublée de la géomorphologie de l'archipel, en fait un ailleurs de prédilection pour les pratiques balnéaires au Québec. La plage et la baignade sont parmi les principales motivations au voyage chez les visiteurs de l'archipel. De plus, pour les visiteurs du Québec (qui sont bon an, mal an les principaux visiteurs de l'archipel), la culture locale unique des îles de la Madeleine en fait une destination qui combine à la fois balnéarité et altérité. Les îles de la Madeleine sont un dépaysement chez soi, une altérité dans un contexte globalement familier.

Le contexte pandémique dans les îles de la Madeleine a augmenté ce sentiment d'altérité, l'archipel ayant été isolé du reste de la province pendant plusieurs semaines lors de la première vague de COVID-19 tout en ayant été relativement épargné par celle-ci. Mentionnons que l'accès y a été limité aux résidents et aux travailleurs essentiels. La levée du confinement et la mise en place du corridor sanitaire pour permettre la mobilité terrestre entre le Québec et le traversier de Souris à l'île-duPrince-Édouard ont permis aux visiteurs d'avoir de nouveau accès à l'archipel. La situation épidémiologique des îles de la Madeleine a créé une image de milieu sûr, à l'opposé des quartiers densément peuplés des centres urbains. Cette image a d'ailleurs été renforcée par le fait qu'assez rapidement, l'archipel a retrouvé une certaine forme de normalité alors que le reste du Québec devait, de son côté, revivre deux autres confinements, dont un caractérisé par l'instauration d'un couvre-feu qui, à l'instant où nous écrivons ces lignes, en est à son cinquième mois de reconduction.

Ce contexte sanitaire propre à une grande partie de la province a conféré à l'archipel une aura particulièrement attractive, et ce malgré les contraintes liées à la traversée des provinces maritimes. De ce fait, les Madelinots ont évité de quitter leur archipel au cours de la saison estivale. Cela a son importance, car il est à noter qu'une partie du modèle de développement touristique de l'archipel se base sur l'hébergement chez l'habitant, qui conduit certains résidents à louer leur propre maison durant la saison estivale au moment où, eux, prennent des vacances à l'extérieur de l'archipel. L'année 2020 a donc vu une cohabitation et un partage inédits des ressources touristiques à plus grande échelle, incluant la population résidente. C'est ainsi que les pratiques de loisirs des résidents dans leur archipel, habituellement concentrées hors de la haute saison touristique de l'été, se sont retrouvées concomitantes de celles de visiteurs. La situation a, tout comme en Gaspésie, induit une dissonance entre différents imaginaires. L'exemple du VTT (appelé quad en Europe francophone) sur les plages et dans les dunes illustre de manière aiguë cette situation. Alors que cette pratique n'est pas promue en tant que produit touristique pour les visiteurs venant de l'extérieur, entre autres du fait de son impact sur des milieux fragiles et de sa difficile cohabitation avec les pratiques balnéaires traditionnelles, le VTT est une activité prisée localement et fortement encadrée par les acteurs territoriaux. Toutefois, à l'été 2020, le contexte de cohabitation entre les visiteurs intéressés par les activités balnéaires et les résidents en VTT a mené à des conflits de valeurs et d'imaginaires plus aigus qu'à la normale, comme en témoignent des images tirées des médias (cf. figure 3). 
Figure 3. Rencontre des imaginaires

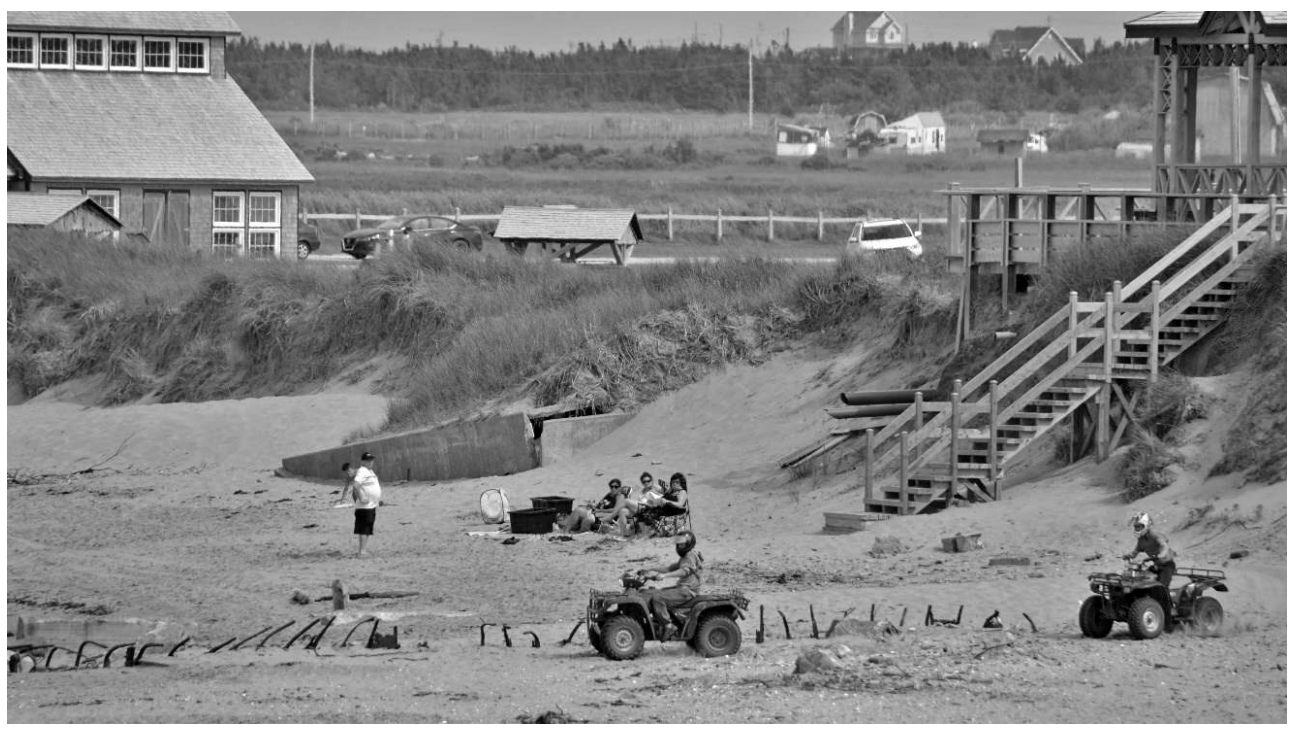

Source : municipalité des îles-de-la-Madeleine

Nous pouvons ainsi observer que, dans les deux cas, le littoral est un espace de vie et de récréation à la fois pour les visiteurs et les résidents. Du côté de la Gaspésie, l'imaginaire touristique de la plage est, en temps normal, beaucoup plus près des pratiques et des représentations des populations résidentes. Néanmoins, dans le contexte particulier de l'été 2020, celui-ci s'est vu malmené par une appropriation rapide et non planifiée des plages suivant des imaginaires balnéaires habituellement très peu présents, voire absents du territoire gaspésien. Du côté des îles de la Madeleine, l'imaginaire balnéaire est déjà bien ancré et a conservé son attrait pour les visiteurs. Toutefois, la superposition de celui-ci aux pratiques de loisirs des résidents a révélé que cet imaginaire balnéaire n'est pas nécessairement partagé à l'identique par la population résidente. Par ailleurs, le "compromis» touristique, selon lequel les impacts et les pressions touristiques sont acceptables en retour de retombées économiques et sociales à même de soutenir l'habitation des territoires périphériques, particulièrement dans le contexte de distance et de dispersion (Dugas, 1980) de l'Estdu-Québec, a été maintenu dans le cas des îles de la Madeleine, le tourisme étant la deuxième industrie de l'archipel et principalement sous contrôle local. Du côté gaspésien, le «compromis touristique " s'est plutôt fissuré : même si les visiteurs ont allégrement investi les littoraux peu aménagés, ils se sont beaucoup moins attardés dans les structures commerciales de la péninsule, celles-ci ayant à composer avec des restrictions de fréquentation conduisant à une réduction de $50 \%$ minimum de leur capacité. De plus, un appel de la santé publique pour que les voyageurs interrégionaux limitent leurs contacts à destination a motivé un ravitaillement au départ plutôt qu'à destination. Un hôtelier témoigne d'ailleurs qu'il n'avait jamais vu autant de clients arriver avec leur propre nourriture dans des glacières portables. 


\section{Développement touristique et reconfigurations des territorialités périphériques}

\section{Localisme}

Alors que la pandémie et les restrictions sanitaires ont obligé les organisations touristiques à se retrancher sur les clientèles domestiques, elles les ont également poussés à retisser leurs activités au sein de relations de proximité (Lapointe, 2020 ; Marcotte et al., 2020). C'est ainsi que nous avons pu voir des entreprises touristiques s'inscrire dans des activités à destination des populations résidentes. Toutefois, dans les premières phases du déconfinement, suite à la première vague de la COVID-19, des territoires périphériques relativement épargnés par la pandémie ont exprimé le désir du maintien de l'isolement, voire d'un repli sur soi, face à une menace considérée comme externe, voire urbaine. Dans un second temps, le redémarrage de l'activité touristique, avec un impact sanitaire limité, a remis en avant les systèmes touristiques locaux. Ce sont effectivement ces réseaux de relations, formels d'une part, avec la mobilisation des membres des associations touristiques régionales, et informels d'autre part, qui ont été mobilisés comme capital pour permettre une reprise de l'activité et l'adaptation de l'offre. Ceux-ci sont aussi devenus l'interface capable de redéfinir localement le rapport à la menace pandémique et à l'importance des gestes barrières maintenus à l'échelle locale.

Ceci a été particulièrement effectif en ce qui concerne les communautés autochtones, qui ont pris en charge la gestion de leur communauté en limitant l'accès des visiteurs à leur espace de vie (Renaud, 2020b), geste qui s'inscrit dans la perspective de leur ambition d'autodétermination territoriale. Dans les communautés allochtones, des gestes de rupture ont également été remarqués. Par exemple, dans les premiers temps de la pandémie, les autorités municipales n'ont pas hésité à gérer l'arrivée des visiteurs à l'aéroport local qui, normalement, est sous juridiction fédérale. Du côté de la Gaspésie, suite au débordement de touristes sur le littoral à l'été 2020 précédemment décrit, les autorités municipales de Gaspé ont demandé à pouvoir réglementer l'accès aux plages, habituellement sous juridiction provinciale. Le gouvernement a donné son accord au printemps 2021 et la municipalité de Gaspé a pu mettre en place une série de mesures qui permettra d'éviter des problèmes similaires à l'été 2021. Ainsi, la municipalité sera de nouveau en droit de mettre des infractions et de mettre en place des aires de campings d'appoint supervisés le long du littoral.

\section{Globalisme}

Alors que la mobilité internationale, comme capital mobilisable des individus, n'a pas encore retrouvé son ampleur prépandémique (alors que de son côté la mobilité internationale des biens et des marchandises a globalement été peu affectée par la crise), la mobilité à l'échelle infranationale s'est, elle, redéployée. C'est ainsi que les espaces lisses du tourisme international et leur capacité à recoder les lieux et les pratiques qui s'y déroulent se sont mis à lisser des espaces striés par la pandémie, non sans frictions (Tzanelli, 2021). En effet, le Worldmaking associé au globalisme touristique s'est mis en œuvre. L'exemple des imaginaires balnéaires génériques superposés à l'imaginaire distinctif des plages non aménagées de la Gaspésie en est un bon exemple. 
De plus, la représentation des espaces urbains densément peuplés en tant qu'espaces à haut risque sanitaire a amené une partie des citoyens mobiles de ces pôles urbains à vouloir se prémunir face à d'éventuels confinements : est alors apparue, pour eux, la nécessité de s'approprier foncièrement des espaces périphériques comme gage de sécurité - reproduisant ainsi les mouvements de population hygiénistes et sanitaires du $\mathrm{XIX}^{\mathrm{e}}$ siècle-, tout en maintenant leur capacité de mobilité pour y accéder. Un phénomène similaire a poussé certains citoyens à se rendre "à tout prix " dans les stations balnéaires du Sud à l'hiver 2021 afin de se « ressourcer », faisant fi des risques de propagation du virus, ce qui a amené le gouvernement canadien à demander aux compagnies aériennes du pays de fermer leurs liaisons vers les destinations du soleil. Toujours en vigueur, cette mesure s'est couplée à une quarantaine obligatoire à l'hôtel, aux frais des voyageurs arrivant de l'international. Désormais privée de cette mobilité internationale et des vacances qu'elle permet habituellement, une frange des citoyens des pôles urbains risque de se rabattre à nouveau sur les imaginaires balnéaires du Québec et chercher à sortir des villes pour profiter "par défaut » des grands espaces des régions périphériques.

\section{Stagnation/fluidité}

Il est encore trop tôt pour statuer sur la sédimentation de ces pratiques de mobilité induites par la pandémie. Alors que la vaccination progresse, mais que le virus mute, nous ne savons pas encore si le processus d'immunisation, caractérisé par ce ballet entre fermetures et ouvertures des espaces et de la société civile (Lapointe, Sarrasin et Lagueux, 2020), durera longtemps. Les nouvelles configurations des territoires du Québec que l'on voit poindre à mi-chemin de la deuxième année de cette pandémie ne seront peut-être qu'un hoquet viral, mais il est important de considérer ce phénomène dans la mesure où cette situation pourrait devenir une nouvelle normalité.

Dans ce contexte, nous pouvons observer une reconfiguration des territorialités du tourisme en écho aux mesures sanitaires. Ainsi émerge une société fluide et mobile, qui reproduit des corridors lisses pour combler non seulement ses besoins de loisirs, par le tourisme, mais également pour s'adapter aux restrictions sanitaires, en se donnant un accès à différents lieux pour travailler, incluant des lieux normalement associés au tourisme et aux vacances. Dans ce contexte, la fluidité recadre des pratiques globalisantes à des échelles infranationales, et ce, sans que les pratiques touristiques ne perdent leur essence. En effet, cette société fluide superpose ses pratiques et imaginaires aux espaces qu'elle fréquente, allant même jusqu'à redéfinir les relations de proximité qui s'y déroulent. Reste que la fluidité n'est pas accessible à l'ensemble de la population, dont une partie est condamnée à la stagnation, entre autres, du fait de la disparition de leurs gagne-pains, car non dématérialisables, associée à la redéfinition de leurs espaces de vie en tant qu'espaces à risques sanitaires.

À l'interface de la stagnation et de la fluidité se trouvent les travailleurs dits " essentiels ", qui gardent un accès à la mobilité, mais dans les instants privilégiés du temps hors travail, tout en étant relativement non mobiles par la nature de leurs activités essentielles, fortement localisées. Ceux-ci sont à la fois ceux qui offrent les services de première ligne à l'ensemble de la population et ceux qui permettent de soutenir la mobilité fluide. Cette position, à l'articulation de la stagnation et de la fluidité, s'inscrit non seulement dans une perspective sociale, mais également 
territoriale. À ce titre, revenons à l'exemple de la ville de Cabano, que nous avons évoqué précédemment vu son rôle comme ultime ville de ravitaillement avant la traversée des Maritimes vers les îles de la Madeleine; celle-ci est exemplaire de l'articulation stagnation/fluidité. Depuis dix ans, cette petite ville industrielle spécialisée dans la transformation des produits de la forêt est en restructuration économique et affiche des velléités touristiques, entre autres par la mise en place d'un parc national et par le développement de villégiatures au bord du lac Témiscouata. Toutefois, ce désir de développement touristique s'est peu concrétisé jusqu'à la mise en place du corridor sanitaire vers les îles de la Madeleine. C'est ainsi qu'un espace en stagnation situé à la liminalité de l'espace touristique québécois est, du jour au lendemain, devenu un lieu essentiel pour permettre le déplacement vers l'espace recherché des îles de la Madeleine. De fait, la ville de Cabano à l'été 2020 est, de façon inattendue, devenue un haut lieu de la mobilité touristique québécoise.

\section{Conclusion}

La reconfiguration des pratiques touristiques apparue dans la foulée de la pandémie de COVID-19 est fortement induite par les différentes contraintes à la mobilité dues aux fermetures de frontières nationales et internationales. De ces nouvelles dynamiques est également apparue une restructuration des rapports socioterritoriaux médiatisés par l'interface mobilité/proximité, lesquels participent également à redéfinir les pratiques touristiques en temps de pandémie. Cette restructuration implique en outre de nouveaux rapports centre/périphérie qui montrent que les dynamiques liées au capital de proximité peuvent jouer en faveur d'un espace périphérique plus résilient que le centre face à la perturbation des mobilités. Il s'agit, non pas seulement en termes de dynamiques touristiques, mais aussi en termes de gestion socioterritoriale globale, d'un «moment » porteur d'opportunités pour les périphéries afin de valoriser leur capacité de prise en charge et d'assurer un développement durable qui réponde à leurs aspirations respectives. En d'autres termes, il s'agit ici de mettre en avant les forces du localisme dans un monde global, tout en évitant les écueils extrémistes de la fluidité et de la stagnation.

L'Est-du-Québec est en effet confronté à des défis qui font écho aux enjeux des régions périphériques à l'échelle globale: dévitalisation, extractivisme, désengagement des pouvoirs centraux, exodes, pour ne nommer que quelques-unes des problématiques qui les concernent. Alors que la dématérialisation du travail est porteuse d'opportunités de développement pour les régions, elle ouvre également la porte à une grande fluidité en matière de capital humain induit, par exemple par l'hypermobilité de la main-d'œuvre. Néanmoins, l'engagement socioterritorial des urbains qui s'installent en périphérie pourrait être remis en question par ceux-ci dès lors que la pandémie s'estompera. Par ailleurs, alors que la mobilité touristique infranationale demeure et est même favorisée en temps de pandémie, la mobilité des populations périphériques, elle, demeure précaire alors que les systèmes de transports locaux, aériens et routiers, sont régulièrement menacés de coupure, lorsqu'ils ne sont pas tout simplement abolis, comme l'a été le transport ferroviaire de passagers en Gaspésie.

Il va sans dire que la question de la mobilité s'inscrit dans une dynamique nuancée et modulable, dans laquelle la valeur attachée au capital de mobilité et de proximité se décline différemment selon les rapports centre-périphérie. De fait, il s'agira de 
poursuivre nos observations pour tenter de saisir les subtilités de la reconfiguration des pratiques touristiques. Certaines configurations seront pérennes, alors que d'autres risquent de disparaitre rapidement suivant le succès des mesures d'éradication du virus. Du côté des régions urbaines, les enjeux socioterritoriaux de la pandémie de COVID-19 sont moins liés au tourisme (même si la chute du tourisme a, pour ces régions, des conséquences notables) qu'aux défis sanitaires, et ce, en considérant les innombrables conséquences dans l'ensemble des sphères de la vie - individuelle, familiale et sociale. De son côté, la périphérie québécoise - même si moins touchée par le virus et n'ayant été que sporadiquement et partiellement confinée - reste dépendante de l'industrie touristique et des enjeux liés aux transformations de la mobilité. Pour celle-ci, les pratiques rattachées au tourisme sont ancrées dans le tissu socio-économique et affectent inévitablement la gestion des espaces de vie.

Notre réflexion a cherché à esquisser des pistes de réflexion sur cette imbrication des capitaux liés à la mobilité et à la proximité, tout en considérant l'importance de tirer le maximum de connaissances de la situation actuelle afin d'arrimer des solutions concrètes aux défis actuels et à ceux qui attendent les régions périphériques dans un monde post-COVID.

\section{BIBLIOGRAPHIE}

Zygmunt BAUMAN, Liquid Modernity, Polity Press, 2000.

Alison CAFFYN, « Advocating and Implementing Slow Tourism », dans Tej Vir Singh (dir.), Critical Debates in Tourism, Channel View Publications, 2012, p. 373-379.

Clermont DUGAS, Un pays de distance et de dispersion, PUQ, 1980.

Antonio FERREIRA, Luca BERTOLINI et Petter NÆSS, « Immotility as Resilience? A Key Consideration for Transport Policy and Research », Applied Mobilities, vol. 2, n 1, p. 16-31, 2017 [http:// dx.doi.org/10.1080/23800127.2017.1283121].

Robert FLETCHER, Ivan MURRAY MASS, Macià BLÁZqUEZ-SALOM et Asunción BLANCO-ROMERO, « Tourism, Degrowth, and the COVID-19 Crisis », POLLEN, 2020 [https://politicalecologynetwork.org/ 2020/03/24/tourism-degrowth-and-the-COVID-19-crisis/amp/].

Keith HOLLINSHEAD, Irena ATELJEVIC et Nazia ALI, « Worldmaking Agency-Worldmaking Authority: The Sovereign Constitutive Role of Tourism », Tourism Geographies, vol. 11, n 4, p. 427-443, 2009 [http://dx.doi.org/10.1080/14616680903262562].

Dominic LAPOINTE, « Reconnecting Tourism after COVID-19: The Paradox of Alterity in Tourism Areas », Tourism Geographies, vol. 22, n 3, p. 633-638, 2020 [http://dx.doi.org/ 10.1080/14616688.2020.1762115].

Dominic LAPOINTE, Bruno SARRASIN et Cassiopée BENJAMIN, « Tourism in the Sustained Hegemonic Neoliberal Order ", Revista Latino-Americana de Turismologia, vol. 4, n 1, p. 16-33, 2018 [https:// doi.org/10.34019/2448-198X.2018.v4.13915]. 
Dominic LAPOINTE, Bruno SARRASIN et Jean LAGUEUX, « Gestion, biopolitique et prospective : quels regards pour la suite du monde? », Téoros, vol. 39, n³, 2020 [https://journals.openedition.org/ teoros/8407].

Pascale MARCotTE, Mohamed REDA KHOMSI, Isabelle FALARDEAU, Romain ROULT et Dominic LAPOINTE, « Tourisme et COVID-19 », Téoros, vol. 39, n 3, 2020 [https://journals.openedition.org/teoros/ 7976].

Polyxeni MOIRA, Dimitrios MYLONOPOUlos et Aikaterini KONDOUDAKI, « The Application of Slow Movement to Tourism: Is Slow Tourism a New Paradigm? ", Journal of Tourism and Leisure Studies, vol. 2, n 2, p. 1-10, 2017 [https://doi.org/10.18848/2470-9336/CGP/v02i02/1-10].

Luc RENAUD, «Cruise Tourism Drives Land Speculation and Displacement in Belize ", NACLA Report on the Americas, vol. 52, n 4, p. 442-47, 2020a [http://dx.doi.org/10.1080/10714839.2021.1840177].

LUC RENAUD, « On vous aime beaucoup, mais repassez une autre fois : tourisme autochtone, hospitalité et confinement au temps de la COVID-19 », Téoros, vol. 39, n³, 2020b [https:// journals.openedition.org/teoros/8531].

JD SHADEL, « "Revenge Travel" Is the Phenomenon That Could Bring Back Tourism with a Bang ", The Washington Post, 29 juillet 2020 [https://www.washingtonpost.com/travel/2020/07/29/ revenge-travel-is-phenomenon-that-could-bring-back-tourism-with-bang/].

Rodanthi TZANELl, Frictions in Cosmopolitan Mobilities: The Ethics and Social Practices of Movement across Cultures, Edward Elgar Publishing, 2021.

Gil VIRY, Emmanuel RAVALET et Vincent KAUfMANN, « High Mobility in Europe: An Overview », dans Gil Viry et Vincent Kaufmann (dir.), High Mobility in Europe: Work and Personal Life, Palgrave Macmillan, 2015, p. 29-58.

\section{NOTES}

1. https://tourismexpress.com/nouvelles/saison-record-en-tourisme-a-montreal

2. https://www.quebec-cite.com/sites/otq/files/media/document/ Bilan\%20annuel\%202020_0.pdf https://www.lesoleil.com/affaires/tourisme-lete-de-reve-se-poursuit-engaspesie-8c524964da721801272db623e5564d82

4. https://www.tourisme-gaspesie.com/images/Upload/medias/comm_bilan_saison_ete2020.pdf 5. En effet, les mesures sanitaires ont obligé les entreprises d'hébergement à diminuer leur capacité d'accueil, parfois jusqu'à $50 \%$. Dans ce contexte, le taux d'occupation est calculé parfois sur la capacité d'accueil complète, d'autre fois sur la capacité d'accueil tronquée.

6. https://www.tourismeilesdelamadeleine.com/fr/medias/actualites/2020/09/30/bilan-desaison-2020/

7. http://veilletourisme.s3.amazonaws.com/2020/09/COVID-V3-faits-saillants-DIFFUSION.pdf

8. La distance entre Cabano et Souris est de $737 \mathrm{~km}$ - à titre de comparaison, la distance ParisMarseille est de $775 \mathrm{~km}$.

9. https://www.lapresse.ca/voyage/quebec-et-canada/2021-01-05/ete-record-a-la-sepaq.php 


\section{RÉSUMÉS}

Suite à la fermeture de leurs frontières avec les États-Unis et l'Est du Canada, les Québécois à la recherche de destinations balnéaires se sont rabattus sur la région maritime de l'Est-du-Québec. En s'appuyant sur les notions de mobilité et de proximité en tant que capital social, cet article analyse la reconfiguration des flux touristiques qui sont apparus avec la pandémie. Ces reconfigurations ont concrétisé certaines pratiques touristiques principalement existantes dans l'imaginaire touristique, tout en faisant émerger de nouvelles pratiques inattendues. Dans ce contexte, cette région touristique hautement sollicitée a vu apparaître de nouvelles contraintes quant à la gestion des espaces de vie, mais elle a également pris acte d'opportunités de développement territorial.

Following the closure of its borders with the United States and Eastern Canada, Quebec residents in search of seaside destinations have turned their interest toward the maritime region of Eastern Quebec. Using the notions of mobility and proximity as social capital, we analyze the reconfiguration of tourist flows that emerged due to the pandemic. These reconfigurations have confirmed certain tourist practices that mainly exist in the tourist imaginary, while at the same time giving rise to new unexpected practices. In this context, this highly sought-after tourist region has seen the emergence of new management constraints on living spaces, but local actors have also identified new opportunities for territorial development.

\section{INDEX}

Mots-clés : mobilité, espace, périphérie, imaginaire, territoire, balnéaire, pandémie, Covid-19, Québec

Keywords : mobility, space, periphery, imaginary, territory, seaside, pandemic, Covid-19, Quebec

\section{AUTEURS}

\section{LUC RENAUD}

PhD - Géographie

Université du Québec à Montréal, département d'études urbaines et touristiques, groupe de recherche et d'intervention tourisme territoire et société (GRITTS), chaire de recherche UQAM sur les dynamiques touristiques et les relations socioterritoriales renaud.luc[at]uqam.ca

\section{DOMINIC LAPOINTE}

PhD - Développement régional

Université du Québec à Montréal, département d'études urbaines et touristiques, groupe de recherche et d'intervention tourisme territoire et société (GRITTS), chaire de recherche UQAM sur les dynamiques touristiques et les relations socioterritoriales

lapointe.dominic[at]uqam.ca 